\title{
Globular Cluster Formation in Galaxy Mergers
}

\author{
Yuexing Li ${ }^{1,2}$, Mordecai-Mark Mac Low ${ }^{2,1}$, and Ralf S. Klessen ${ }^{3}$ \\ ${ }^{1}$ Columbia Univ, $N Y ;{ }^{2}$ AMNH, NY, USA ${ }^{3}$ AIP, Potsdam, Germany
}

\begin{abstract}
We present preliminary results of a high resolution simulation of globular cluster formation in a galaxy merger using GADGET (Springel et al. 2001). A barotropic equation of state ( $\mathrm{Li}$ et al 2003) is implemented to include effects of cooling and heating. After one orbital period, a dozen proto-globular clusters are identified in the tidal tails.
\end{abstract}

\section{Simulation}

We simulate an equal-mass, head-on merger of two identical disk galaxies moving on parabolic orbits. Each galaxy has a dark matter halo, a stellar disk, and a gas disk (Springel 2000) . The mass of the galaxy is $M_{200}=3 \times 10^{10} M_{\odot}$, of which gas is $10 \%$. The total particle number is $N_{t o t}=1.4 \times 10^{5}$, the mass resolution is $2.3 \times 10^{6} M_{\odot}$, and the spatial resolution is $1 \mathrm{pc}$.
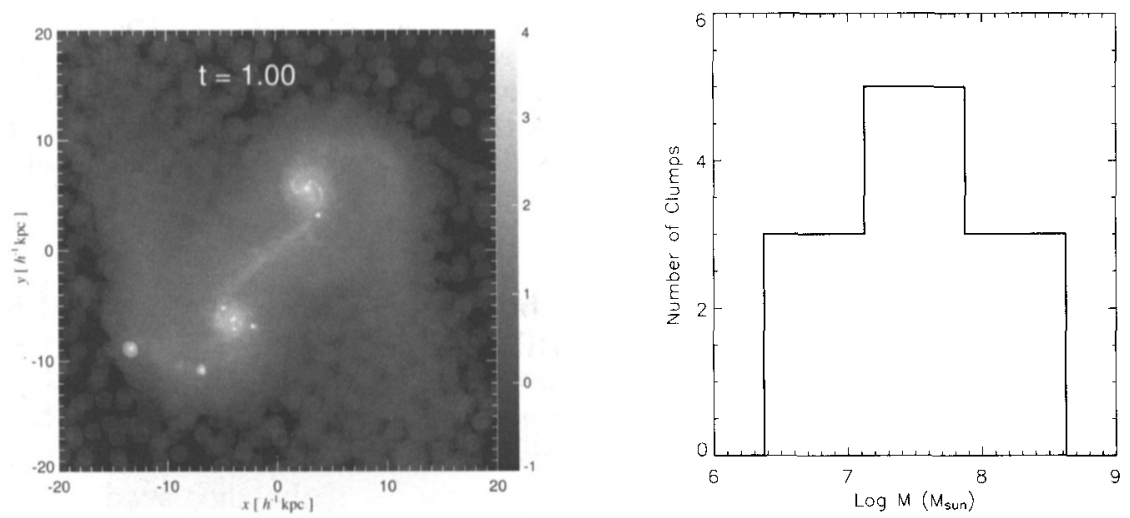

Figure 1. Proto-globular clusters identified in tidal tails and bridges, and their mass histogram derived with a clump-finding algorithm.

Acknowledgments. We acknowledge support from V. Springel, Kade Fellowship, NSF AST99-85392, NASA NAG5-13028, and the Emmy Noether of Deutsche Forschungsgemeinschaft (KL1358/1).

\section{References}

Li, Y., Klessen, R., \& Mac Low, M-M, 2003, ApJ, 592, 975

Springel, V., Yoshida N. \& White, S. 2001, New Astronomy 6, 79

Springel, V. 2000, MNRAS 312, 859 